\title{
Milieu therapy in in-patient treatment for adolescents with anorexia nervosa
}

T. Lebailly ${ }^{1}$, S. Saint-André1.

${ }^{1} \mathrm{CHRU}$ Brest, Service Hospitalo-Universitaire de Psychiatrie de l'Enfant et Adolescent, Bohars, France.

\section{Objectives:}

To discuss the role of in-patient units in the treatment of adolescents with anorexia nervosa. In this frame, to consider how and under what conditions a milieu therapy approach is appropriate and can be efficient in such clinical situations.

\section{Background:}

Anorexia Nervosa is a rare (prevalence around $1 \%$ in France and in developed countries) and complex disease for which no treatment demonstrates superiority over others. Hospitalization for young anorexic, whether it is in pediatric or in child psychiatry units, is problematic as much on its relevance as on its effectiveness. Should we proceed to family separations or, on the contrary, absolutely avoid them? What to do when disorders become chronic or extremely severe?

\section{Materials and Methods:}

This presentation will be based on case studies involving patients hospitalized in the Child and Adolescent psychiatry department of the Brest University Hospital. The treatment of this cases will be compared to the data brought by an international literature review.

\section{Results and Conclusions:}

Our system includes several units, outpatient, pediatric inpatient, child psychiatry inpatient and care\&study unit, which have no differences of values but of propositions. They articulate themselves without superiority, but rather with a temporal gradation. The care provided is thus always individualized in order to work with the patient and his family to solve their relationship problems. The milieu therapy allows the adolescent and his/her family to resume a positive dynamic of their previously frequently frozen interactions. 